\title{
Optical spectropolarimetry observations of the BL Lac-type object PKS 0537-441 after a period of quiescence
}

\author{
J Barnard, ${ }^{a, *}$ B van Soelen, ${ }^{a}$ J Cooper, ${ }^{a} \mathbf{R}$ J Britto, ${ }^{a}$ J P Marais, ${ }^{a}$ I P van der \\ Westhuizen, ${ }^{a}$ D A H Buckley, ${ }^{a, b}$ H M Schutte, ${ }^{c}$ M Böttcher, ${ }^{c}$ B Vaidya, ${ }^{d}$ S Acharya ${ }^{d}$ \\ and A Martin-Carrillo ${ }^{e}$ \\ ${ }^{a}$ Department of Physics, University of the Free State, \\ Bloemfontein, South Africa \\ ${ }^{b}$ South African Astronomical Observatory, \\ Cape Town, South Africa \\ ${ }^{c}$ Center for Space Research, North West University, \\ Potchefstroom, South Africa \\ ${ }^{d}$ Discipline of Astronomy Astrophysics and Space Engineering, Indian Institute of Technology Indore, \\ Indore, India \\ eSpace Science Group, School of Physics, University College Dublin, \\ Dublin, Ireland
}

E-mail: Els]@ufs.ac.za

Blazars are a radio-loud subclass of active galactic nuclei with relativistic jets closely aligned with our line of sight. They are highly variable across all time-scales, and display rapid flares across multiple wavelength bands. At optical wavelengths, the observed emission is a superposition of non-thermal emission arising from the jet, and thermal emission that originates from the accretion disc, broad-line region, dust torus and host galaxy. Optical spectropolarimetry observations of blazars can be used to disentangle the thermal non-polarised and non-thermal polarised emission components in blazar emission during flaring and quiescent states. As part of a long-term monitoring campaign, spectropolarimetry observations have been taken with the Southern African Large Telescope of a selection of blazars during periods of increased activity. Here we focus on spectropolarimetry results and Fermi-LAT light curve of the BL-Lac type object PKS 0537-441 observed around a period of increased activity. It was observed to be active at both optical and gamma-ray energies after recovering from a faint state in late 2018. Spectropolarimetry observations were taken twice, with the degree of linear polarisation being between 35\% and $40 \%$ during the first observation, and between $10 \%$ and $15 \%$ during the second, while there was no significant change in the strength of the emission line.

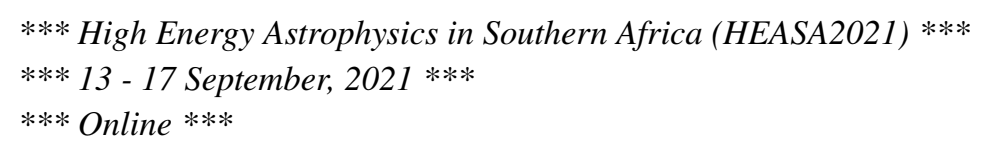

\footnotetext{
${ }^{*}$ Speaker
} 


\section{Introduction}

The central active cores of some galaxies, or active galactic nuclei (AGN), which are powered by the accretion of material onto a central supermassive black hole, display non-thermal, broadband emission across the entire electromagnetic spectrum [1]. Blazars are a radio-loud subclass of AGN with a prominent jet-emission component, as the jet propagates in a direction that is within $\sim 10^{\circ}$ of the observer's line of sight, resulting in highly Doppler boosted emission. Blazar emission is extremely variable on minute up to year time-scales, with large variations observable across multiple wavelength bands [2].

Blazar spectral energy distributions (SEDs) typically consist of two broad, non-thermal components. The lower-energy component spans the radio to optical/UV and sometimes soft X-ray wavelength bands. Two different emission components contribute in the lower-energy component: a non-thermal, polarised component and an underlying thermal, non-polarised component. The non-thermal component is produced by synchrotron emission from the jet, while the underlying thermal emission originates from the accretion disc, dust torus, broad-line region (BLR) and host galaxy itself [3]. The higher-energy component, extending from the X-ray to the gamma-ray wavelength bands, can be reproduced by both leptonic and hadronic models [3].

For flaring blazars, optical polarimetry can serve as a diagnostic tool that separates the thermal and non-thermal contributions from one another. This, in turn, allows for constraints to be placed on various SED- and jet-model parameters [4]. Here we present optical spectropolarimetry observations of a blazar, PKS 0537-441, obtained around a period of increased gamma-ray activity, following a quiescent state [5]. These observations form part of a long-term observing campaign that aims at monitoring the optical spectropolarimetric behaviour of a large selection of blazars.

\section{Observations}

Spectropolarimetry observations were undertaken with the Southern African Large Telescope (SALT) under the long-term transient observing programme 2018-2-LSP-001, that observes various transients, including novae, gamma-ray bursts, cataclysmic variables, and flaring blazars [4]. In the case of blazars, observations are normally triggered by an increase in gamma-ray activity detected by the Fermi Large Area Telescope (LAT). From 2016 April to 2021 May, the transient programme has collected SALT spectropolarimetry observations of 21 different blazars, consisting of 14 Flat-Spectrum Radio Quasars (FSRQs), 6 BL Lac type objects (BLLs), and 1 Blazar Candidate of Uncertain Type (BCU). In the following we limit the discussion to one BLL observed during a period of increased activity as it was recovering from a faint state, namely PKS 0537-441 $(z=0.894)[6]$.

\subsection{Optical Spectropolarimetry}

The SALT observations were performed using the Robert Stobie Spectrograph (RSS) in spectropolarimetry LINEAR mode to measure the degree of linear polarisation [7, 8]. Data reduction was performed using a modified version of the POLSALT reduction pipeline, ${ }^{1}$ which allows the wave-

\footnotetext{
${ }^{1}$ https://github.com/saltastro/polsalt.git
} 


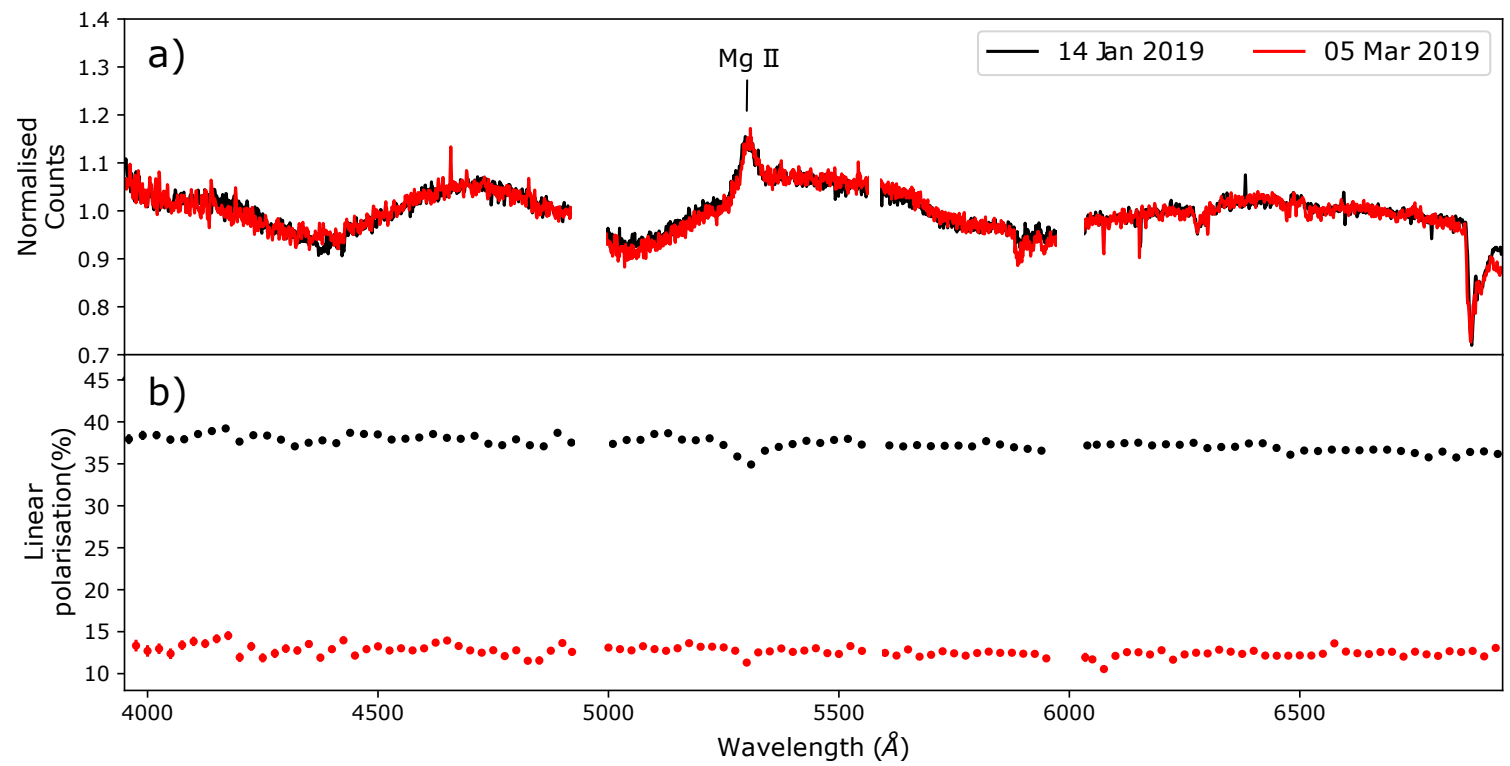

Figure 1: Spectropolarimetry observations of PKS 0537-441 taken around a period of increased activity after quiescence, where (a) is the normalised counts, and (b) the observed degree of linear polarisation.

length calibration to be performed using the IRAF/NOAO package. ${ }^{2}$ After the wavelength calibration, the modified pipeline performs additional cosmic-ray cleaning using the LACOSMIC package. ${ }^{3}$ The spectra in Figure 1 show two empty regions due to the chip gaps, and a smaller gap at $\sim 5600 \AA$ due to the removal of a strong skyline.

The spectropolarimetry observation of the BLL was performed using the pg0900 grating at an angle of $14.375^{\circ}$, covering a wavelength range from $4500 \AA$ to $7500 \AA$ (with a resolving power of $R=870-1200$ ). For wavelength calibration, the Argon arc lamp was used.

\subsection{Gamma-ray Observations}

The Fermi-LAT gamma-ray light curves were obtained from the Fermi-LAT Light Curve Repository (LCR). ${ }^{4}$ The data for PKS 0537-441 was obtained with a 3-day binned data cadence, within an energy range of $0.1-100 \mathrm{GeV}$. The minimum detection significance, or upper limit, was set at a Test Statistic (TS) value of $4(\approx 2 \sigma)$, and the spectral fitting was done with a free photon index.

\section{Results}

Figure 1 shows the spectropolarimetry results of PKS 0537-441 after the target has recovered from a quiescent state (since 2018 December 13, MJD 58465) and showed activity in both optical and gamma-ray since [5]. The degree of linear polarisation during the first observation was between $35 \%$ and $40 \%$. Seven weeks later, the degree of polarisation was observed to have decreased to between

${ }^{2} \mathrm{http} / / /$ ast.noao.edu/data/software

${ }^{3}$ https://github.com/larrybradley/lacosmic.git

${ }^{4}$ https://fermi.gsfc.nasa.gov/ssc/data/access/lat/LightCurveRepository/ 


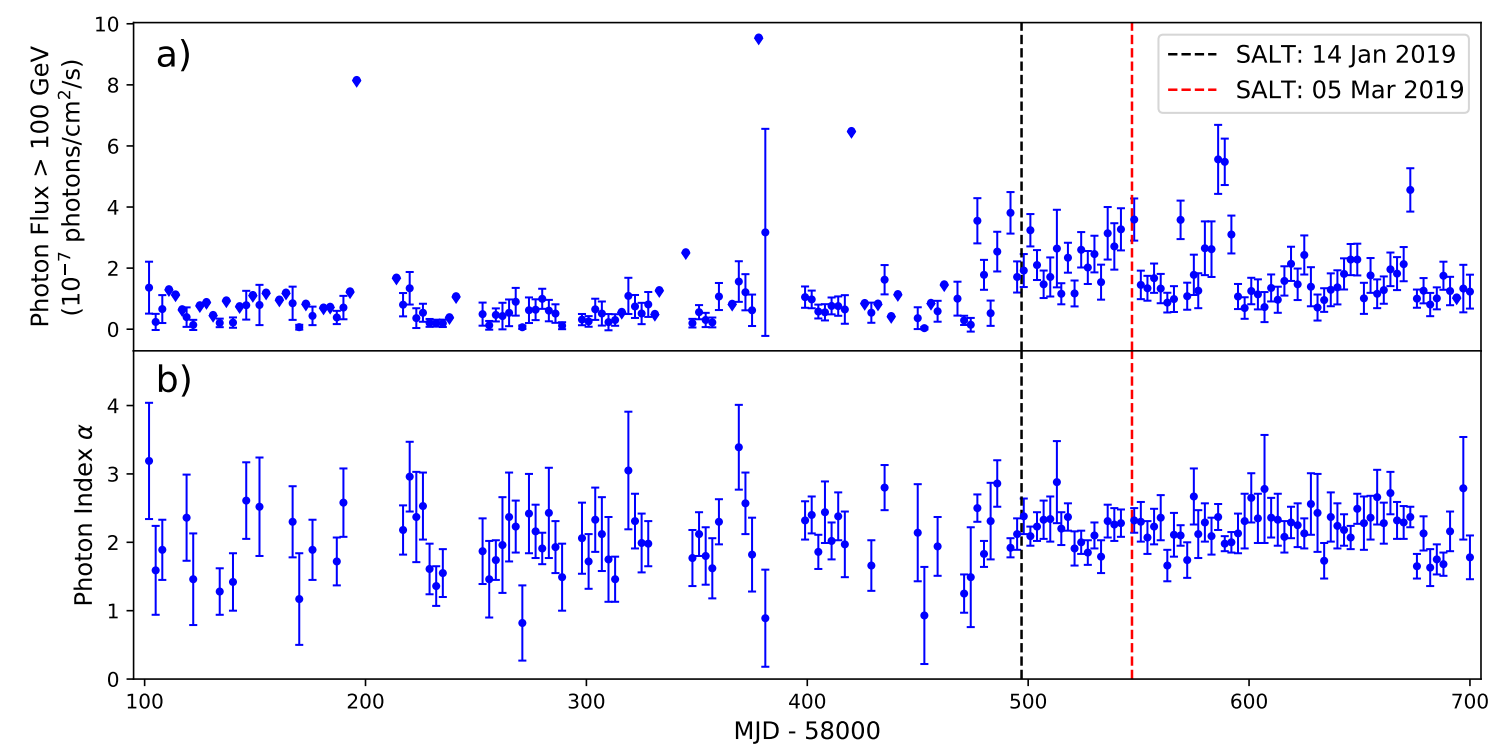

Figure 2: The Fermi-LAT light curve (a), and photon spectral index (b) for PKS 0537-441. The flux was measured at $E>100 \mathrm{GeV}$ in 3-day bins, spanning the period of 2017 December 15 to 2019 August 05 (MJD 58102 to 58700). The dashed lines in (a) indicate the dates at which the SALT spectropolarimetry observations were taken.

$10 \%$ and $15 \%$, thus showing substantial variability. The Fermi-LAT light curve (Figure 2) spanning a period of 2017 December - 2019 August clearly shows the transition from a quiescent to an active state, increasing from an average photon flux $(E>100 \mathrm{GeV})$ of below $1 \times 10^{-7}$ photons cm $\mathrm{cm}^{-2} \mathrm{~s}^{-1}$ to a varying flux of up to six times higher. On 2019 January 14 (MJD 58497), the gamma-ray photon flux $(E>100 \mathrm{GeV})$ reached $(1.92 \pm 0.54) \times 10^{-7}$, and reached $(3.36 \pm 0.771) \times 10^{-7}$ photons cm $^{-2} \mathrm{~s}^{-1}$ on 2019 March 05 (MJD 58547). Hence, there is an increase in the gamma-ray photon flux while a decrease in optical polarisation is observed.

\section{Discussion and Conclusion}

For the BLL, PKS 0537-441, SALT spectropolarimetry observations were performed during a period of increased gamma-ray and optical activity following a period of quiescence (see Figure 1). It can be seen from Figure 1 that the degree of linear polarisation has decreased between the two observations. The gamma-ray light curve (Figure 2) obtained from the Fermi LCR show the increased gamma-ray activity during the period in question. This trend is in agreement with what is known about blazar jets: non-thermal synchrotron emission in the jet increases due to turbulence in jet's magnetic field, and therefore increases degree of linear polarisation. Hence, polarisation studies can link directly to the jet's magnetic field structure and can place constraints on model parameters.

These observations form part of a long-term monitoring campaign that aims at contemporaneous, multi-wavelength observations of blazars during both flaring and quiescent states in an attempt 
to gain a better understanding of blazar jets, the jet's magnetic field, the state of the accretion discs, as well as the nature of the electron population in the jet.

\section{Acknowledgments}

All of the spectropolarimetry observations reported in this paper were obtained with the Southern African Large Telescope (SALT) under program 2018-2-LSP-001 (PI: DAH Buckley). B. van Soelen acknowledges support by the National Research Foundation of South Africa (Grant Number: 116300)

\section{References}

[1] Padovani P 2017 Front. Astron. Space Sci. 4 12-18

[2] Beckmann V and Shrader, C R 2012 Active Galactic Nuclei (Weinheim: Wiley)

[3] Böttcher M, Reimer A, Sweeney K and Prakash A 2013 ApJ 768

[4] Böttcher M, van Soelen B, Britto R J, Buckley D A H, Marais J P and Schutte H 2017 Galaxies $552-58$

[5] Nesci R and Ojha R 2019 ATel 12357

[6] Mao L S 2011 New Astronomy 168

[7] Burgh E B, Nordsieck K H, Kobulnicky H A, Williams T B, O'Donoghue D, Smith M P and Percival J W 2003 SPIE 4841 1463-71

[8] Kobulnicky H A, Nordsieck K H, Burgh E B, Smith M P, Percival J W, Williams T B and O’Donoghue D 2003 SPIE 4841 1634-44 